\title{
Splicing conservation signals in plant long noncoding RNAs
}

\author{
JOSE ANTONIO CORONA-GOMEZ, ${ }^{1}$ IRVING JAIR GARCIA-LOPEZ, ${ }^{1}$ PETER F. STADLER, ${ }^{2,3,4,5,6,7}$ \\ and SELENE L. FERNANDEZ-VALVERDE ${ }^{1}$

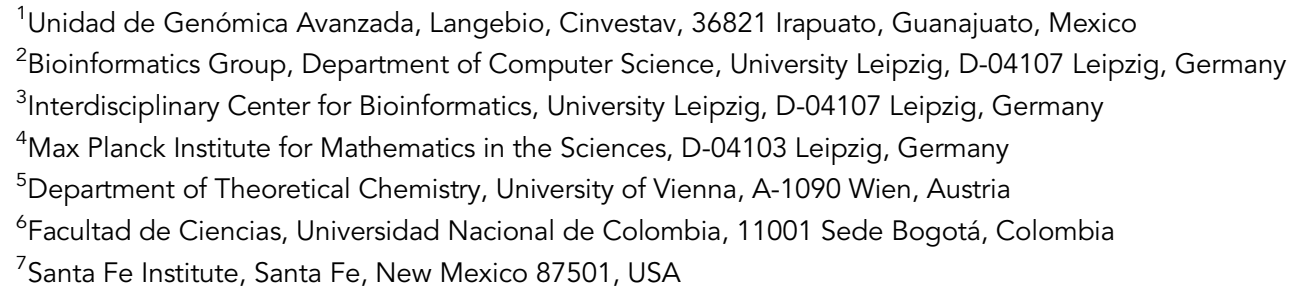

\section{ABSTRACT}

Long noncoding RNAs (IncRNAs) have recently emerged as prominent regulators of gene expression in eukaryotes. LncRNAs often drive the modification and maintenance of gene activation or gene silencing states via chromatin conformation rearrangements. In plants, IncRNAs have been shown to participate in gene regulation, and are essential to processes such as vernalization and photomorphogenesis. Despite their prominent functions, only over a dozen IncRNAs have been experimentally and functionally characterized. Similar to its animal counterparts, the rates of sequence divergence are much higher in plant IncRNAs than in protein coding mRNAs, making it difficult to identify IncRNA conservation using traditional sequence comparison methods. Beyond this, little is known about the evolutionary patterns of IncRNAs in plants. Here, we characterized the splicing conservation of IncRNAs in Brassicaceae. We generated a whole-genome alignment of 16 Brassica species and used it to identify synthenic IncRNA orthologs. Using a scoring system trained on transcriptomes from $A$. thaliana and $B$. oleracea, we identified splice sites across the whole alignment and measured their conservation. Our analysis revealed that $17.9 \%(112 / 627)$ of all intergenic IncRNAs display splicing conservation in at least one exon, an estimate that is substantially higher than previous estimates of IncRNA conservation in this group. Our findings agree with similar studies in vertebrates, demonstrating that splicing conservation can be evidence of stabilizing selection. We provide conclusive evidence for the existence of evolutionary deeply conserved IncRNAs in plants and describe a generally applicable computational workflow to identify functional IncRNAs in plants.

Keywords: long noncoding RNAs; IncRNA; splice sites; multiple sequence alignments; evolution; conservation; evolutionary plasticity

\section{INTRODUCTION}

Long noncoding RNAs (IncRNAs), by definition, do not code for proteins. Over the last decade, a wide variety of mechanisms have been discovered by which IncRNAs contribute to the regulation of the expression of protein-coding genes and small RNAs (Chekanova 2015; Liu et al. 2015; Ulitsky 2016; Wang and Chekanova 2017; Yamada 2017). Most IncRNAs are found in the nucleus associated with the chromatin, regulating gene expression by recruiting components of the epigenetic machinery to specific genomic locations. Some IncRNAs also influence genome stability and nuclear domain organization. Serving as molecular sponges and decoys, they act both at the transcrip-

Corresponding authors: studla@bioinf.unileipzig.de, selene. fernandez@cinvestav.mx

Article is online at http://www.rnajournal.org/cgi/doi/10.1261/rna. 074393.119. tional level, by affecting RNA-directed DNA methylation; in post-transcriptional regulation, by inhibiting the interaction between microRNAs (miRNAs) and their target messenger RNAs (mRNAs); and by controlling alternative splicing due to sequestration of splicing factors (Bardou et al. 2014). Hence, they differ not only in size but also in their biogenesis and molecular mechanisms from small RNAs such as miRNAs and siRNAs (Bánfai et al. 2012). IncRNAs are regulated and processed similar to mRNAs (Mercer and Mattick 2013) and their expression patterns are often very specific to particular tissues or developmental stages (Mercer and Mattick 2013). Recent data suggest

(C) 2020 Corona-Gomez et al. This article is distributed exclusively by the RNA Society for the first 12 months after the full-issue publication date (see http://rnajournal.cshlp.org/site/misc/terms.xhtml). After 12 months, it is available under a Creative Commons License (Attribution-NonCommercial 4.0 International), as described at http:// creativecommons.org/licenses/by-nc/4.0/. 
that there appears to be a distinction between highly conserved, constitutively transcribed IncRNAs and tissuespecific IncRNAs with low expression levels (Deng et al. 2018b; Sarropoulos et al. 2019).

Despite their often very poor sequence conservation (Necsulea et al. 2014), the majority of IncRNAs are wellconserved across animals, as evidenced by the conservation of many of their splice sites (Nitsche et al. 2015). While well-conserved as entities, they show much more plasticity in their gene structure and sequence than protein-coding genes. The many lineage-specific differences have implicated IncRNAs as major players in lineagespecific adaptation (Lozada-Chávez et al. 2011): Changes in transcript structure are likely associated with the inclusion or exclusion of sets of protein or miRNA binding sites and hence may have large effects on function and specificity of a particular IncRNA.

The systematic annotation of orthologous IncRNAs is important not only to provide reasonably complete maps of the transcriptome, but also as a means of establishing that a particular IncRNA has a biological function. After all, conservation over long evolutionary timescales is often used as the most important argument for the biological function of an open reading frame in the absence of direct experimental evidence for translation and experimental data characterizing the peptide product. While a large amount of work is available showing that vertebrate genomes contain a large number of secondary elements that are under negative selection (Smith et al. 2013; Hezroni et al. 2015; Nitsche and Stadler 2017; Seemann et al. 2017) and the majority of human IncRNAs are evolutionary old (Nitsche et al. 2015), a much less systematic and complete picture is available for plants.

In fact, detailed studies into the evolution of plant IncRNAs have been rare until very recently. An analysis of IncRNAs in five monocot and five dicot species (Deng et al. 2018b) found that the majority of IncRNAs are poorly conserved at sequence level while a majority is highly divergent but syntenically conserved. These positionally conserved IncRNAs were previously found to be located near telomeres in A. thaliana (Mohammadin et al. 2015). Plant IncRNAs have also been shown to display canonical splicing signals (Deng et al. 2018b). Another study in 10 Brassicaceae genomes found $22 \%$ conservation of intergenic IncRNA loci (Nelson et al. 2016), as well as little evidence of an impact of whole-genome duplications or transposable element (TE) activity on the emergence of lincRNAs.

Nevertheless, there are some plant IncRNAs whose regulatory functions have been studied extensively and are understood at a level of detail comparable to most proteins (Rai et al. 2019): COOLAIR in Brassicaceae has a crucial role in the vernalization process (Hawkes et al. 2016) and its transcription accelerates epigenetic silencing of the flowering locus C (FLC) (Rosa et al. 2016). The
IncRNA HID1 is a key component in promoting photomorphogenesis in response to different levels of red light (Wang et al. 2014). HID1 is highly conserved and acts through binding to chromatin in trans to act upon the PIF3 promoter. A similar trans-acting IncRNA is ELENA1, which functions in plant immunity (Mach 2017). Competing endogenous RNAs (ceRNAs) acts as "sponges" for miRNAs. In plants, ceRNAs are a large class of IncRNAs (Yuan et al. 2017; Paschoal et al. 2018) and form extensive regulatory networks (Meng et al. 2018; Zhang et al. 2018). The paradigmatic example in A. thaliana is IPS1, which sequesters miR399, resulting in changes in phosphate homeostasis (Franco-Zorrilla et al. 2007).

Although the functional characterization of plant IncRNAs is confined to a small number of cases, plant IncRNAs are being reported at a rapidly increasing pace (Nelson et al. 2016). As in the case of animals, it is important therefore to amass evidence for the functionality of individual transcripts. Differential expression, or correlations with important regulatory proteins or pathways alone do not provide sufficient evidence to decide whether a transcript has a causal effect or whether its expression pattern is a coincidental downstream effect. As a first step toward prioritizing candidates for functional characterization, we advocate for the use of unexpected deep conservation of the gene structure as an indicator of biological function. While logically this still does not inform about function in a specific context, it is much less likely that changes in expression patterns of a conserved and thus presumably functional molecule are without biological consequence.

The much higher level of plasticity in plant genomes, compared to animal genomes, potentially makes it more difficult to trace the evolution of IncRNAs. We therefore concentrate here on a phylogenetically relatively narrow group, the Brassicaceae, with genomes that are largely alignable with each other. We track the conservation of functional elements, in particular splice junctions, through the entire data set. This provides direct evidence also in cases where transcriptome data is not available in sufficient coverage and or sufficient diversity of tissues and/or developmental stages. As a final result, we provide a list of homologous IncRNAs in Brassicaceae as well as a detailed map of the conservation of splice sites in this clade.

\section{RESULTS}

\section{Identification of splice sites and IncRNAs}

To build a $A$. thaliana splice junction reference, we identified about 125,000 introns using the transcriptomes of Liu et al. (2012) compared with 175,000 introns annotated in TAIR10 (Release 38) (Berardini et al. 2015). The smaller number was expected as (i) only introns with convincing coverage by uniquely mapping reads were considered 
and (ii) not all A. thaliana genes are expressed in these four transcriptomes. Consistent with previous reports (Brown et al. 1996; Hebsgaard 1996), the vast majority of the detected splice junctions have the canonical GT/AG motif required for inclusion into our splice site map. In total, we identified 222,772 individual sites in A. thaliana $(117,644$ donor and 121,002 acceptor sites). 55\% of all donors but only $13 \%$ of the acceptors have aligned sequences in the WGA (Supplemental Fig. S3). In addition, many splice sites have evidence of expression in transcriptome data from other species (Supplemental Table S3).

To characterize splicing conservation in IncRNAs, we focused solely on intergenic long noncoding RNAs (lincRNAs). Conservation of splice sites in IncRNAs overlapping with coding genes may be confounded by the coding gene conservation signal, resulting in false positives. The IncRNAs described by Liu et al. (2012) comprise 595 lincRNAs with predicted introns, with only 18 with confirmed introns as annotated in Araport9 (Liu et al. 2012), while in Araport11 (Cheng et al. 2017) 288 annotated lincRNAs out of 2444 have introns. We also used an additional set of IncRNAs expressed in A. thaliana cotyledons and hypocotyls in Col-0 plants in normal light or shade conditions (Kohnen et al. 2016). These libraries were stranded, and had three replicates as well as sufficient depth to produce a high confidence IncRNA annotation. As these transcriptomes are only derived from two experimental conditions (shadow and light) (Kohnen et al. 2016), they encompass only a fraction of the IncRNAs expressed throughout the $A$. thaliana life cycle. We identified 2375 IncRNA transcripts, 1465 of which overlapped with protein coding RNAs, while 808 were found in intergenic regions and were thus considered bona fide lincRNAs. In our analysis, we found 159 lincRNAs that were included in neither Araport11 nor TAIR10 (Berardini et al. 2015; Cheng et al. 2017). Furthermore, we excluded all IncRNAs that had any overlap with other annotated ncRNAs thus depleting our set of IncRNAs that may be microRNA or snoRNA precursors, as small RNAs are generally conserved. All 808 lincRNAs transcripts aggregated in 627 lincRNA genes, of which 58 have multiple isoforms. In contrast to the situation in animals, lincRNAs are therefore mostly mono-exonic in A. thaliana. Of the 627 lincRNA genes, only 173 had at least one intron and thus were used to test splice site conservation in IncRNAs; of these 173, only 35 were previously annotated in the Araport11 database.

\section{Conservation of IncRNAs}

To identify conserved elements by position, we extracted aligned sequences corresponding to different annotations sets from the WGA. Between $69.6 \%$ to $44.2 \%$ of the $A$. thaliana genome was aligned with other Brassicaceae species. For the protein-coding genes annotated in Araport11 (Cheng et al. 2017), the alignment recovery rate ranges from $95.3 \%(26,153 / 27,445)$ (A. lyrata) to $86.9 \%(23,856)$ $27,445)$ (Aethionema arabicum). As expected, the values are substantially lower for the Araport11 lincRNAs, where we recover between $77.1 \%(1885 / 2444)$ in $A$. lyrata and $50.8 \%(1243 / 2444)$ in A. arabicum. Using our own annotation, we recover between $62.0 \%$ (389/627) in A. lyrata and $38.1 \%(239 / 627)$ in A. arabicum, i.e., values comparable to the overall coverage of the genome. This reflects the fact that IncRNA sequences experience very little constraint on their sequence. Conservation (as measured by alignability) is summarized in Figure 1 for different types of RNA elements. These values are comparable to a previous estimate of $\sim 22 \%$ of the lincRNA loci are at least partially conserved at the sequence level in the last common ancestor of Brassicaceae (Nelson et al. 2016).

Conservation of splice sites is a strong indication for the functionality of the transcript. In order to evaluate splice site conservation quantitatively, we constructed a splicing map that identifies for every experimentally determined splice site the homologous position in the other genomes and evaluates them using the MES (see Materials and Methods for details). Figure 2 shows the splicing map for the lincRNA TCONS00053212-00053217 as an illustrative example. Despite the unusually complex transcript structure and the conservation throughout the Brassicaceae, so far nothing is known about the function of this lincRNA. While not all splice sites are represented in all species in the WGA, almost all MES values in this lincRNA are well above the threshold of MES $>0$. This contrasts with a random sampling of splice sites in coding and noncoding regions in all genomes in the WGA (Supplemental Fig. S4). Indeed, the probability of identifying a random splice site with an MES value greater than 0 in $A$. thaliana is 0.0237 (acceptor) and 0.0165 (donor) for coding genes, and 0.0225 (acceptor) and 0.0168 (donor) for lincRNAs (Supplemental Fig. S4). Most of this lincRNA isoforms therefore can be expected to be present throughout the Brassicaceae, even though the locus is not annotated in Ensembl Plants (release 42) for B. oleracea, B. rapa, and A. lyrata. Only the short first exon and the $5^{\prime}$ most acceptor of the last exon are poorly conserved by sequence even in close relatives of $A$. thaliana.

In order to validate the predicted lincRNA splice sites, we investigated publicly available RNA-seq data from eight of the species included in this study (Supplemental Table S2). The depth of these data varied considerably. We therefore compared the fraction of recovered IncRNA predictions with the fraction of mRNAs that were detectable in the same RNA-seq data (Supplemental Table S4). As expected, we observed that the relative validation rate increases with the depth of data, presumably owing to the fact that IncRNAs are on average less highly expressed and more specifically expressed than mRNAs. Nevertheless, the validation rate in our data of lincRNAs is on average $33.3 \%$ in the eight species used for validation 


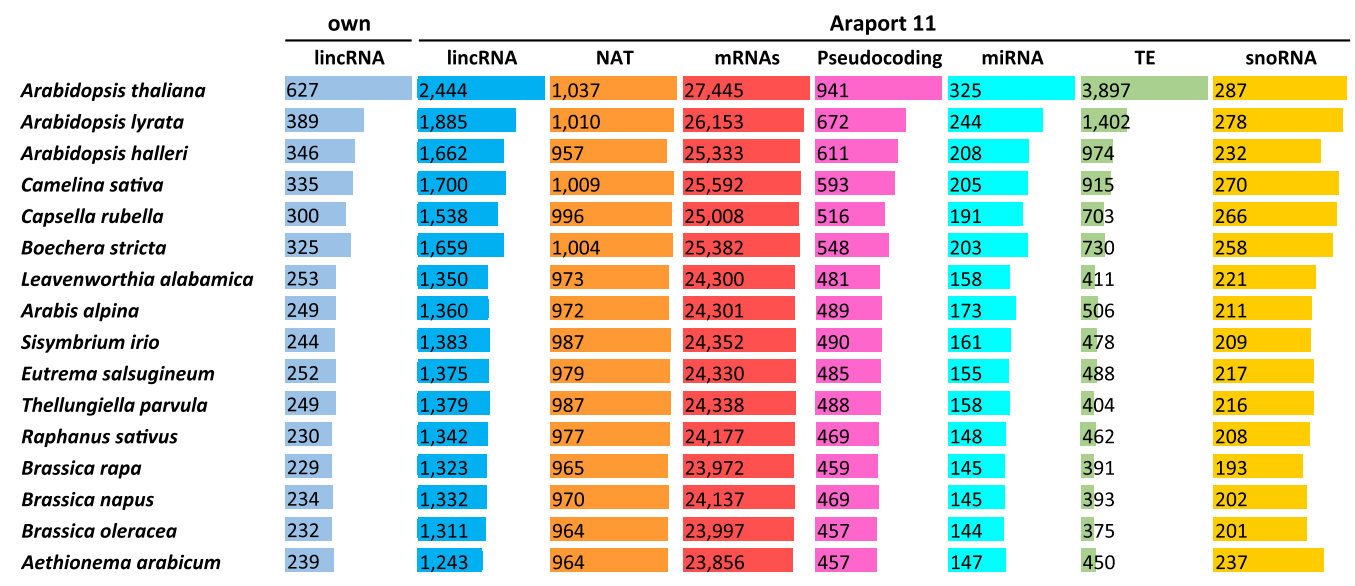

FIGURE 1. Conservation of genes by position in WGA. Own: lincRNAs genes expressed in shade experiments (Kohnen et al. 2016). Araport11 database annotations (Cheng et al. 2017): lincRNAs (long intergenic noncoding RNAs), NAT (Natural antisense transcripts), Coding genes (messenger RNAs), miRNA (microRNAs), Pseudocoding (Pseudocoding genes), TE (Transposable elements), snoRNA (Small nucleolar RNAs)

and $10.7 \%$ for lincRNAs in Araport11 (Cheng et al. 2017), while for coding genes it is $57.5 \%$.

On a genome-wide scale, the conservation of splice sites in lincRNAs provides a lower bound on the fraction of lincRNAs that are under selective constraint as a transcript. We find that 112 of the 173 spliced A. thaliana lincRNAs have at least one conserved splice site in another species (Fig. 3).

As expected, we find that splice sites in lincRNAs are much less well conserved than splice sites in protein coding genes (Fig. 4). In total, we identified 39 lincRNAs conserved between the most distant species and $A$. thaliana and 26 lincRNAs with conservation in at least one splice site in the 16 species included in the WGA. These numbers are much lower than for coding genes. Albeit this is expected, given the high conservation of protein coding genes, one has to keep in mind that coding genes on average have at least six introns (Deng et al. 2018b), hence it is much more likely to observe conservation of at least one splice site and in lincRNAs with only one or two introns (see Fig. 3).

The potential incompleteness of annotated IncRNAs, for example, due to low expression levels, is of concern in this context. It has little influence on our conclusions, however, since incomplete or fragmented annotation only causes us to underestimate the depth of conservation: We might occasionally miss the best-conserved splice junction and we might count fragments as independent, less conserved IncRNAs. Unrecognized overlap with known short ncRNAs is of little concern because the latter are almost never spliced. The only exception are the "splice-siteoverlapping" SO-microRNAs (Mattioli et al. 2014), which, however, are almost exclusively found in coding genes (Pianigiani et al. 2018) and thus removed by our filters. We therefore assume that such artefacts have a very minor impact in our analysis.
In comparison to vertebrates, we observe a much lower level of conservation as measured by gene structure. For instance, $35.2 \%$ of the transcripts are conserved between human and mouse (Nitsche et al. 2015), while between A. thaliana and $A$. arabicum we only find splice site conservation in $6.2 \%(39 / 627)$ of our own lincRNAs and 1.3\% (32/2444) of lincRNAs annotated in Araport11. This difference is even more striking given the fact that the evolutionary distance between human and mouse ( 75 Mya) (Waterston et al. 2002) is larger than between A. thaliana and A. arabicum ( 54 Mya) (Beilstein et al. 2010).

Transposable elements (TEs) are important factors in IncRNA origin (Kapusta et al. 2013). To explore if conserved lincRNAs may be related to TEs, we compared our 627 lincRNAs with the genomic positions of TEs described in Araport11 database. We find only 149 of 627 lincRNAs overlap with TEs and these lincRNAs display significantly lower positional conservation than other lincRNAs in the WGA. Indeed, only 11 were found to be positionally conserved between $A$. thaliana and B. rapa. The number of TEs which coincide with lincRNAs with conserved splice sites is even smaller; of the 173 lincRNAs with introns only 11 overlapped with TEs. From all 3897 TEs in the Araport 11 database, only 450 are conserved by position in the WGA between $A$. thaliana and $A$. arabicum. This represents only $11.5 \%$ of the TEs, that is, less than the percentage of the lincRNAs conserved by genomic position (Fig. 1).

\section{DISCUSSION}

In this work we explore the conservation of IncRNAs in the Brassicaceae plant family and we find conservation at different levels: From 627 lincRNAs identified we have $38.1 \%(239 / 627)$ conserved by genomic position as determined by the presence of alignable sequence. A small 
A

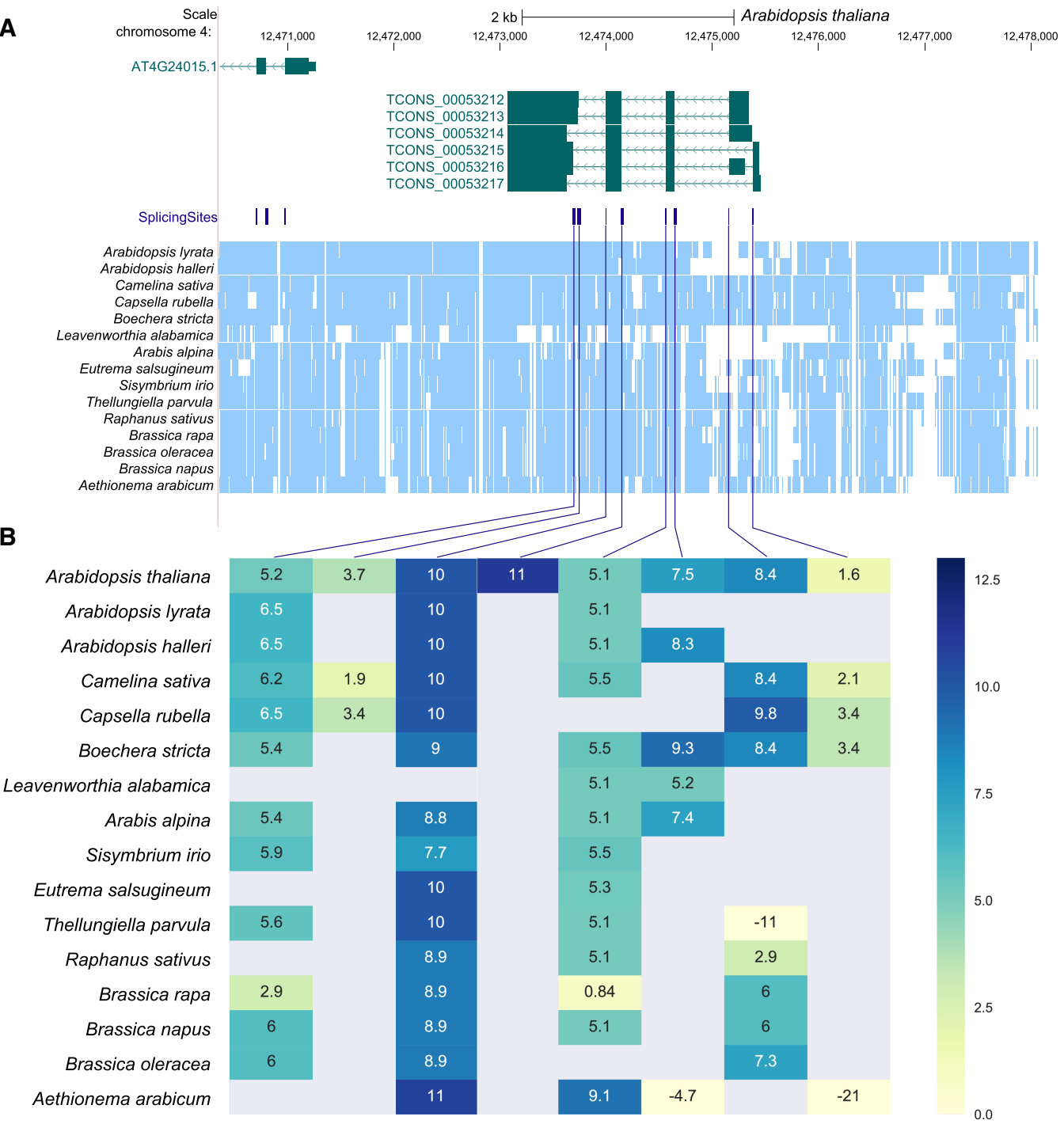

FIGURE 2. Splicing conservation map of lincRNA locus TCONS00053212-TCONS00053217. (A) UCSC Genome browser screenshot of the TCONS00053212-TCONS00053217 locus; blocks denote exons, and line with arrows, introns. The arrow direction indicates direction of transcription. Splicing sites are shown in purple. Light blue blocks represent aligned regions as identified by Cactus. (B) Heatmap of TCONS00053212TCONS00053217 MES in each splice site (columns) in each species (rows), linked to its position in A with a purple line. MES are shown from more negative (light yellow) to more positive (dark blue). MES values $>0$ were used to identify conserved splice sites.

fraction (27.6\%) of these lincRNAs contain introns. Only $19.1 \%$ of spliced lincRNAs are conserved between A. thaliana and $B$. oleracea, the species with the lowest level of conservation in our data set. While sequence conservation may be a consequence of selective constraints on DNA elements, conservation of splice sites directly indicates selective constraints at the transcript level, and thus can be interpreted as evidence for an (unknown) functional role of the lincRNA. The 112 lincRNAs with conserved splice sites are therefore attractive candidates for studies into IncRNA function.

In spite of the small number of spliced lincRNAs analyzed, we find most of them (nearly 65\%) have at least one conserved splice site. This is substantially higher than estimates of conservation by sequence of about 22\% amongst Brassica species (Nelson et al. 2016). Thus there is a stronger evolutionary constraint on plant lincRNA processing as measured by splice site conservation than by sequence. This is similar to what was previously found in placental mammals (Nitsche et al. 2015), where $\sim 70 \%$ of the IncRNAs have splice site conservation. However, this level of conservation should be considered lower given the divergence time between placental mammals is larger than the divergence times between the Brassicaceae analyzed in this study (52.6 Mya [Kagale et al. 2014]). At least in part this difference is the consequence of the prevalence of single-exon lincRNAs in this clade and the small number of splice sites in those 

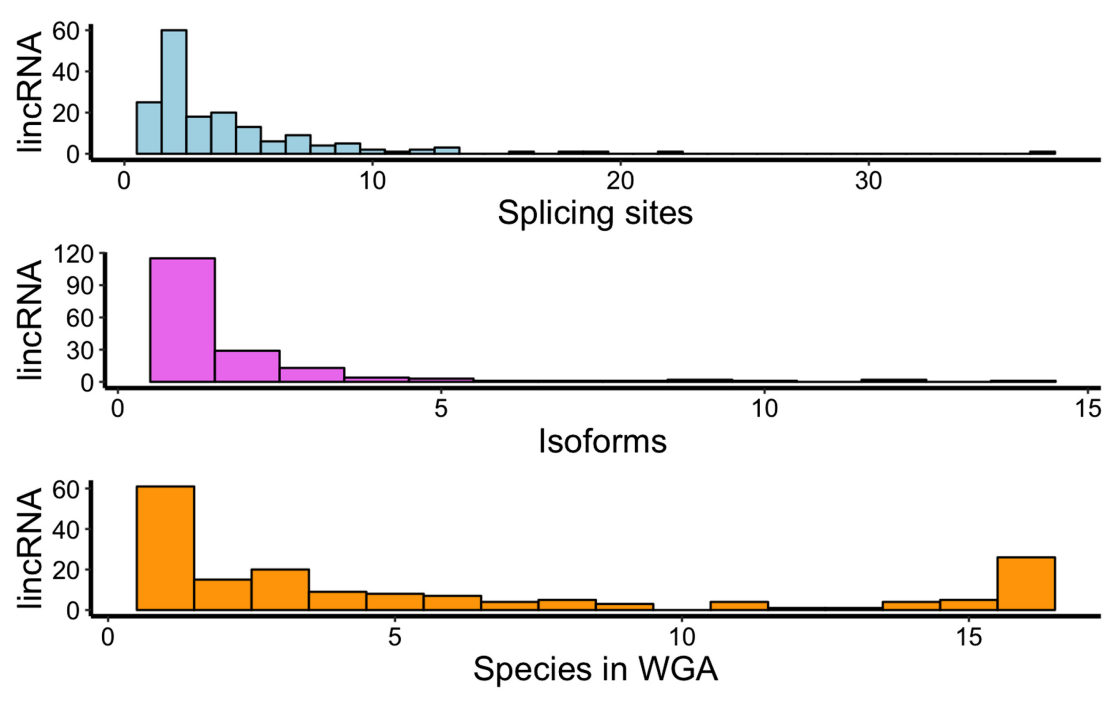

FIGURE 3. Histograms showing number of splicing sites, isoforms, and conservation in WGA of the 173 lincRNAs genes with introns in Own data set. Blue bars indicate the number of splicing sites per lincRNA gene with introns. Purple bars visualize the number of isoforms by lincRNA gene. Orange bars refer to the number of species in which lincRNA genes are conserved.

lincRNAs that contain introns. This reduced the power of the method we used to detect splice site conservation, and hints at a reduced importance of introns in the small genomes of the Brassicaceae. The apparent lower conservation of splice sites may also result from our decision to use $A$. thaliana as a reference which, in addition to having a drastically reduced genome, may have also been subjected to clade-specific intron-loss. Transcriptomes of other Brassicas and other plant families that have not undergone drastic genome reduction will help clarify the actual prevalence on monoexonic and intron-gain -loss in plant IncRNAs.

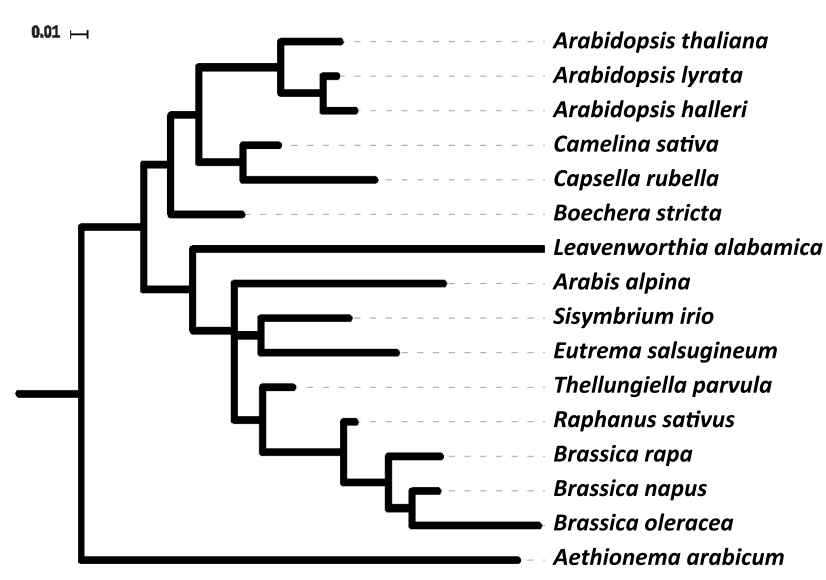
lincRNAs associated with TEs, compared to a much stronger association in Poaceae (Wang et al. 2017). We interpret this to be a consequence of the substantial reduction of genome size in Brassicas. More detailed comparisons of lincRNA conservation with other plant families will have to await better assembled and annotated genomes to construct adequate WGAs.

A limitation of our work is the restriction to intergenic IncRNAs, caused by the need to avoid potential overlaps of the splice sites with other constrained elements. High quality transcriptomes from diverse tissues for most species could alleviate this shortcoming, allowing us to

FIGURE 4. Conservation genes in the Brassicaceae family measured by the conservation of splice sites. (Blue) Own lincRNA set (627); (green) lincRNAs in Araport11 (2444); (red) coding RNA genes $(27,445)$. Only genes with at least one intron are shown. Phylogenetic tree scale is in changes per site. 
construct splicing maps using only experimental evidence. Spurious sequence conservation would then no longer influence the results. This is of particular relevance in Brassicaceae, since $\sim 70 \%$ of transcripts have antisense IncRNAs (Wang et al. 2014). These had to be excluded from our analysis even though at least some of them, for example, COOLAIR (Hawkes et al. 2016), are known to have important biological functions. Complementarily to the analysis of splice site conservation, conserved RNA secondary structure can serve as evidence of selection constraints at the RNA level (Washietl et al. 2005). Moreover, structural analysis can be applied to both spliced and monoexonic transcripts. So far, no genomewide assessment of conservation of RNA secondary structure has been reported for plants. However, recent structurome sequence data indicates that RNA structure is also under selection at the genome-wide level in plants (Deng et al. 2018a).

In summary we showed here that higher plants contain at least dozens and most likely hundreds of well-conserved-and with near certainty functional-long noncoding RNAs. We provide an initial catalog of candidates for more detailed exploration, in many cases supported by direct evidence for expression in several species. We furthermore contribute a generic workflow that can be used to uncover conserved IncRNAs in other groups of plants. Given the rapidly expanding collection of publicly available RNA-seq data sets, we suggest that a comparative analysis of IncRNA conservation can complement standard procedures for genome annotation and thus eventually lead to a comprehensive picture of IncRNA diversity and evolution in plants.

\section{MATERIALS AND METHODS}

\section{Whole-genome alignment}

We selected sixteen plant genomes from those available for the Brassicaceae family in NCBI, Phytozome, and Ensembl-Plants (Supplemental Table S1) based on the quality of assembly, as measured by the number of contigs/scaffolds. All genomes were downloaded in fasta format. Mitochondrial and chloroplast sequences were excluded based on annotation.

The genomes were aligned using Cactus v0 (Paten et al. 2011). Like other whole-genome alignments (WGA) methods, Cactus v0 uses small regions with very high sequence similarity as anchors. To resolve conflicts at this level, Cactus v0 uses a specialized graph data structure that produces better overall alignments than other WGA approaches (Earl et al. 2014). The final WGA result was stored in HAL format (Hickey et al. 2013) for further processing.

\section{Transcriptome data and assembly}

We used four previously published base-line transcriptomes for $A$. thaliana (Liu et al. 2012) (GEO accession number GSE38612), as well as transcriptomes of shade response experiments from Kohnen et al. (2016) (GEO accession number GSE81202). For Brassica oleracea we used transcriptomes from Yu et al. (2014) (Expression Atlas accession number E-GEOD-42891). To validate predicted IncRNAs, we used the publicly available transcriptome data sets listed in Supplemental Table S2. All transcriptomes were downloaded as raw reads in fastq format.

We generated our own IncRNA annotation using all single-end stranded sequencing libraries from Kohnen et al. (2016). Libraries were quality-filtered using Trimmomatic v0.32 (Bolger et al. 2014), and mapped to the TAIR10 genome (Berardini et al. 2015) using TopHat v2.1.1 (Trapnell et al. 2009) with parameters: -I 20 -| 1000 -read-edit-dist 3 -read-realign-edit-dist 0 -library-type fr-firstsrand -g 1. Transcripts were assembled with Cufflinks v2.2.1 (Trapnell et al. 2010) with parameters: -overlap-radius 1 -p 8 -l 1000 -min-intron-length 20 -g TAIR10_GFF3.gff -library-type frfirststrand and subsequently merged into a single reference transcriptome using Cuffmerge v2.2.1.

\section{IncRNA annotation}

LncRNAs in the (Kohnen et al. 2016) data set were annotated using two independent methods. First, coding and noncoding transcripts were identified with CPC v0.9.r2 (Coding Potential Calculator) (Kong et al. 2007), a support vector machine classifier. Additionally, we used a strict stepwise annotation workflow (Cabili et al. 2011) on all transcripts. Specifically, we removed transcripts $<200 \mathrm{nt}$ in length and identified ORFs 75 aminoacids or longer. Identified ORFs were compared against the NCBI non redundant (nr) database using blastx v2.2.31 and blastp v2.2.31 (Altschul et al. 1990) with E-value and cutoff of $<10$ for a sequence to be considered potentially coding. In addition, we used HMMER v3.1b2 (Wheeler and Eddy 2013) to search for Pfam protein domains, signalP v4.1 (Petersen et al. 2011) to identify signal peptides, and tmhmm v2.0 (Krogh et al. 2001) for transmembrane helices. Only sequences that had no similarity with proteins in $\mathrm{nr}$ and no identifiable protein domains, signal peptides or transmembrane domains were annotated as bona fide IncRNAs.

To characterize the genomic context of identified IncRNAs, we used bedtools v2.25.0 (Quinlan and Hall 2010) and compared the IncRNA annotation with the protein coding gene annotation in Araport11 (Cheng et al. 2017). All IncRNA candidates that overlapped a coding sequence or some other ncRNA (miRNA, snoRNA, snRNA) by at least $1 \mathrm{nt}$ were discarded.

\section{Splicing map}

The construction of splicing maps requires a seed set of experimentally determined splice sites in at least one species as well as a statistical model to assess the conservation of splice donors and splice acceptors whenever no direct experimental evidence is available.

To obtain these data for Brassicaceae, we mapped the reference transcriptomes to the corresponding reference genome using STAR v2.4.0.1 (Dobin et al. 2013) with default parameters. The table of splice junctions produced by STAR v2.4.0.1 for each data set were concatenated. Only splice junctions that (a) had at least 10 uniquely mapped reads crossing the junction, and (b) showed the canonical GT/AG dinucleotides delimiting the intron (c) within 
an intron of size between $59 \mathrm{bp}$ and $999 \mathrm{bp}$ were retained for subsequent analyses. Since some of the transcriptome data sets were not strand-specific, we included CT/AC delimiters, interpreting these as reverse-complements. The same procedure was used for splice site validation in other species, where each transcriptome was mapped against their respective genomes prior to splice junction identification. See Supplemental Table S1 for accessions.

For each identified splice site in $A$. thaliana, we used the HalTools v2.1 liftover tool (Hickey et al. 2013) to determine the corresponding orthologous positions in all other genome sequences in the Cactus v0 generated WGA. For each of the retained splice sites, we extracted the genomic sequences surrounding the donor and acceptor sites. If more than one homolog per species is contained in the WGA, we retained the candidate with the highest sequence similarity to $A$. thaliana. For each known splice site and their orthologous position, the MaxEntScan v0 splice-site score (MES) (Yeo and Burge 2004) was computed with either the donor or acceptor model provided the region contained neither gaps nor ambiguous nucleotides (Supplemental Fig. S1). Otherwise, the regions were treated as nonconserved. MaxEntScan v0 models sequence motifs with a probabilistic model based on the Maximum Entropy Principle, which considers adjacent and nonadjacent dependencies between positions. Several works have verified that the MES is an informative score to measure splice site conservation (Eng et al. 2004; Nitsche et al. 2015). A MaxEntScan v0 splice-site score cutoff of 0 was used (Supplemental Fig. S2). This cut-off value was estimated from the distribution of the MES values obtained from $A$. thaliana and B. oleracea transcriptome data (Supplemental Fig. S2). To estimate the rate of false positives, we calculated the probability of finding random splice sites in coding genes and in lincRNAs. For this, we sampled 10,000 random splice positions for both acceptor and donor splicing motifs. In addition to this we calculate the MES values of the same random positions conserved in all WGA species, verifying that they follow the same distribution as in A. thaliana. All positively predicted splice-sites, that is, those with MES $>0$, were added to the splicing map. The pipeline implementing this analysis is available at: bitbucket.org/ JoseAntonioCorona/splicing_map_plants.

\section{DATA DEPOSITION}

TrackHubs for all data sets and IncRNAs used in this study as well as WGA are available here: www.bioinf.uni-leipzig.de/ Publications/SUPPLEMENTS/19-001/BrassicaceaeWGA/hub.txt. Additional information and machine readable intermediate results are provided at http://www.bioinf.uni-leipzig.de/ Publications/SUPPLEMENTS/19-001.

\section{SUPPLEMENTAL MATERIAL}

Supplemental material is available for this article.

\section{ACKNOWLEDGMENTS}

This work was funded in part by Consejo Nacional de Ciencia y Tecnologia (CONACYT PhD Scholarship 338379 [J.A.C.-G.],
CONACYT Research Fellowship 2015-72223 [S.L.F.-V.]), the Deutsche Forschungsgemeinschaft (DFG) grant no. STA850/191 to P.F.S., and by a Royal Society Newton Advanced Fellowship (NAF\R1\180303) awarded to S.L.F.-V. We are grateful to Thomas Gatter for advice on transcriptome assembly.

Received December 17, 2019; accepted March 28, 2020.

\section{REFERENCES}

Altschul SF, Gish W, MillerW, Myers EW, Lipman DJ. 1990. Basic local alignment search tool. J Mol Biol 215: 403-410. doi:10.1016/ S0022-2836(05)80360-2

Bardou F, Ariel F, Simpson CG, Romero-Barrios N, Laporte P, Balzergue S, Brown JWS, Crespi M. 2014. Long noncoding RNA modulates alternative splicing regulators in Arabidopsis. Dev Cell 30: 166-176. doi:10.1016/j.devcel.2014.06.017

Bánfai B, Jia H, Khatun J, Wood E, Risk B, Gundling WE, Kundaje A, Gunawardena HP, Yu Y, Xie L, et al. 2012. Long noncoding RNAs are rarely translated in two human cell lines. Genome Res 22: 1646-1657. doi:10.1101/gr.134767.111

Beilstein MA, Nagalingum NS, Clements MD, Manchester SR, Mathews S. 2010. Dated molecular phylogenies indicate a Miocene origin for Arabidopsis thaliana. Proc Natl Acad Sci 107: 18724-18728. doi:10.1073/pnas.0909766107

Berardini TZ, Reiser L, Li D, Mezheritsky Y, Muller R, Strait E, Huala E. 2015. The Arabidopsis information resource: making and mining the 'gold standard' annotated reference plant genome. Genesis 53: 474-485. doi:10.1002/dvg.22877

Bolger AM, Lohse M, Usadel B. 2014. Trimmomatic: a flexible trimmer for Illumina sequence data. Bioinformatics 30: 2114-2120. doi:10 .1093/bioinformatics/btu170

Brown JWS, Smith P, Simpson CG. 1996. Arabidopsis consensus intron sequences. Plant Mol Biol 32: 531-535. doi:10.1007/ BF00019105

Cabili MN, Trapnell C, Goff L, Koziol M, Tazon-Vega B, Regev A, Rinn JL. 2011. Integrative annotation of human large intergenic noncoding RNAs reveals global properties and specific subclasses. Genes Dev 25: 1915-1927. doi:10.1101/gad.17446611

Charles M, Tang H, Belcram H, Paterson A, Gornicki P, Chalhoub B. 2009. Sixty million years in evolution of soft grain trait in grasses: emergence of the softness locus in the common ancestor of pooideae and ehrhartoideae, after their divergence from panicoideae. Mol Biol Evol 26: 1651-1661. doi:10.1093/molbev/msp076

Chekanova JA. 2015. Long non-coding RNAs and their functions in plants. Curr Opin Plant Biol 27: 207-216. doi:10.1016/j.pbi.2015 .08 .003

Cheng CY, Krishnakumar V, Chan AP, Thibaud-Nissen F, Schobel S, Town CD. 2017. Araport11: a complete reannotation of the Arabidopsis thaliana reference genome. Plant J 89: 789-804. doi:10.1111/tpj.13415

Deng H, Cheema J, Zhang H, Woolfenden H, Norris M, Liu Z, Liu Q, Yang X, Yang M, Deng X, et al. 2018a. Rice in vivo RNA structurome reveals RNA secondary structure conservation and divergence in plants. Mol Plant 11: 607-622. doi:10.1016/j.molp .2018.01.008

Deng P, Liu S, Nie X, Weining S, Wu L. 2018b. Conservation analysis of long non-coding RNAs in plants. Sci China Life Sci 61: 190-198. doi:10.1007/s11427-017-9174-9

Dobin A, Davis CA, Schlesinger F, Drenkow J, Zaleski C, Jha S, Batut P, Chaisson M, Gingeras TR. 2013. STAR: ultrafast universal RNA-seq aligner. Bioinformatics 29: 15-21. doi:10.1093/bioinformatics/ bts635 
Earl D, Nguyen N, Hickey G, Harris RS, Fitzgerald S, Beal K, Seledtsov I, Molodtsov V, Raney BJ, Clawson H, et al. 2014. Alignathon: a competitive assessment of whole-genome alignment methods. Genome Res 24: 2077-2089. doi:10.1101/gr .174920 .114

Eng L, Coutinho G, Nahas S, Yeo G, Tanouye R, Babaei M, Dörk T, Burge C, Gatti RA. 2004. Nonclassical splicing mutations in the coding and noncoding regions of the ATM gene: maximum entropy estimates of splice junction strengths. Hum Mutat 23: 67-76. doi:10.1002/humu.10295

Franco-Zorrilla JM, Valli A, Todesco M, Mateos I, Puga MI, RubioSomoza I, Leyva A, Weigel D, Garcia JA, Paz-Ares J. 2007. Target mimicry provides a new mechanism for regulation of microRNA activity. Nat Genet 39: 1033-1037. doi:10.1038/ ng2079

Hawkes EJ, Hennelly SP, Novikova IV, Irwin JA, Dean C, Sanbonmatsu KY. 2016. COOLAIR antisense RNAs form evolutionarily conserved elaborate secondary structures. Cell Rep 16: 30873096. doi:10.1016/j.celrep.2016.08.045

Hebsgaard S. 1996. Splice site prediction in Arabidopsis thaliana premRNA by combining local and global sequence information Nucleic Acids Res 24: 3439-3452. doi:10.1093/nar/24.17.3439

Hezroni H, Koppstein D, Schwartz MG, Avrutin A, Bartel DP, Ulitsky I. 2015. Principles of long noncoding RNA evolution derived from direct comparison of transcriptomes in 17 species. Cell Rep 11: 1110-1122. doi:10.1016/j.celrep.2015.04.023

Hickey G, Paten B, Earl D, Zerbino D, Haussler D. 2013. HAL: a hierarchical format for storing and analyzing multiple genome alignments. Bioinformatics 29: 1341-1342. doi:10.1093/ bioinformatics/btt128

Kagale S, Robinson SJ, Nixon J, Xiao R, Huebert T, Condie J, Kessler D, Clarke WE, Edger PP, Links MG, et al. 2014. Polyploid evolution of the Brassicaceae during the cenozoic era. Plant Cell 26: 2777-2791. doi:10.1105/tpc.114.126391

Kapusta A, Kronenberg Z, Lynch VJ, Zhuo X, Ramsay LA, Bourque G, Yandell M, Feschotte C. 2013. Transposable elements are major contributors to the origin, diversification, and regulation of vertebrate long noncoding RNAs. PLoS Genet 9: e1003470. doi:10 .1371/journal.pgen.1003470

Kohnen MV, Schmid-Siegert E, Trevisan M, Petrolati LA, Sénéchal F, Müller-Moulé P, Maloof J, Xenarios I, Fankhauser C. 2016. Neighbor detection induces organ-specific transcriptomes, revealing patterns underlying hypocotyl-specific growth. Plant Cell 28: 2889-2904. doi:10.1105/tpc.16.00463

Kong L, Zhang Y, Ye ZQ, Liu XQ, Zhao SQ, Wei L, Gao G. 2007. CPC: assess the protein-coding potential of transcripts using sequence features and support vector machine. Nucleic Acids Res 35: 345349. doi:10.1093/nar/gkm391

Krogh A, Larsson B, Von Heijne G, Sonnhammer ELL. 2001. Predicting transmembrane protein topology with a hidden Markov model: application to complete genomes. J Mol Biol 305: 567-580. doi:10.1006/jmbi.2000.4315

Liu J, Jung C, Xu J, Wang H, Deng S, Bernad L, Arenas-Huertero C, Chua NH. 2012. Genome-wide analysis uncovers regulation of long intergenic noncoding RNAs in Arabidopsis. Plant Cell 24: 4333-4345. doi:10.1105/tpc.112.102855

Liu J, Wang H, Chua NH. 2015. Long noncoding RNA transcriptome of plants. Plant Biotechnol J 13: 319-328. doi:10.1111/pbi.12336

Lozada-Chávez I, Stadler PF, Prohaska SJ. 2011. "Hypothesis for the modern RNA world": a pervasive non-coding RNA-based genetic regulation is a prerequisite for the emergence 2 of multicellular complexity. Orig Life Evol Biosphys 41: 587-607. doi:10.1007/ s11084-011-9262-1

Mach J. 2017. The long-noncoding RNA Elena1 functions in plant immunity. Plant Cell 29: 916. doi:10.1105/tpc.17.00343.
Mattioli C, Pianigiani G, Pagani F. 2014. Cross talk between spliceosome and microprocessor defines the fate of pre-mRNA. Wiley Interdiscip Rev RNA 5: 647-658. doi:10.1002/wrna.1236

Meng X, Zhang P, Chen Q, Wang J, Chen M. 2018. Identification and characterization of ncRNA-associated ceRNA networks in Arabidopsis leaf development. BMC Genomics 19: 607. doi:10 .1186/s12864-018-4993-2

Mercer TR, Mattick JS. 2013. Structure and function of long noncoding RNAs in epigenetic regulation. Nature 20: 300-307. doi:10 $.1038 / \mathrm{nsmb} .2480$

Mohammadin S, Edger PP, Pires JC, Schranz ME. 2015. Positionallyconserved but sequence-diverged: identification of long non-coding RNAs in the Brassicaceae and Cleomaceae. BMC Plant Biol 15: 217. doi:10.1186/s12870-015-0603-5

Necsulea A, Soumillon M, Warnefors M, Liechti A, Daish T, Zeller U, Baker JC, Grützner F, Kaessmann H. 2014. The evolution of IncRNA repertoires and expression patterns in tetrapods. Nature 505: 635-640. doi:10.1038/nature 12943

Nelson AD, Forsythe ES, Devisetty UK, Clausen DS, Haug-Batzell AK, Meldrum AM, Frank MR, Lyons E, Beilstein MA. 2016. A genomic analysis of factors driving lincRNA diversification: lessons from plants. G3(Bethesda) 6: 2881-2891. doi:10.1534/g3.116.030338

Nitsche A, Stadler PF. 2017. Evolutionary clues in IncRNAs. Wiley Interdiscip Rev RNA 8: 14-17. doi:10.1002/wrna.1376

Nitsche A, Rose D, Fasold M, Reiche K, Stadler PF. 2015. Comparison of splice sites reveals that long noncoding RNAs are evolutionarily well conserved. RNA 21: 801-812. doi:10.1261/rna.046342.114

Paschoal AR, Lozada-Chávez I, Domingues DS, Stadler PF. 2018. ceRNAs in plants: computational approaches and associated challenges for target mimics research. Brief Bioinformatics 19: 12731289. doi:10.1093/bib/bbx058

Paten B, Earl D, Nguyen N, Diekhans M, Zerbino D, Haussler D. 2011. Cactus: algorithms for genome multiple sequence alignment. Genome Res 21: 1512-1528. doi:10.1101/gr.123356.111

Petersen TN, Brunak S, von Heijne G, Nielsen H. 2011. SignalP 4.0: discriminating signal peptides from transmembrane regions. Nat Methods 8: 785-786. doi:10.1038/nmeth.1701

Pianigiani G, Licastro D, Fortugno P, Castiglia D, Petrovic I, Pagani F. 2018. Microprocessor-dependent processing of splice site overlapping microRNA exons does not result in changes in alternative splicing. RNA 24: 1158-1171. doi:10.1261/rna.063438.117

Quinlan AR, Hall IM. 2010. BEDTools: a flexible suite of utilities for comparing genomic features. Bioinformatics 26: 841-842. doi:10.1093/bioinformatics/btq033

Rai MI, Maheen A, Lightfoot DA, Gurha P, Afzal AJ. 2019. Classification and experimental identification of plant long non-coding RNAs. Genomics 111: 997-1005. doi:10.1016/j.ygeno.2018.04.014

Rosa S, Duncan S, Dean C. 2016. Mutually exclusive sense-antisense transcription at FLC facilitates environmentally induced gene repression. Nat Commun 7: 13031. doi:10.1038/ncomms13031

Sarropoulos I, Marin R, Cardoso-moreira M, Kaessmann H. 2019. Developmental dynamics of IncRNAs across mammalian organs and species. Nature 571: 510-514. doi:10.1038/s41586-0191341-x

Seemann SE, Mirza AH, Hansen C, Bang-Berthelsen CH, Garde C, Christensen-Dalsgaard M, Torarinsson E, Yao Z, Workman CT, Pociot $F$, et al. 2017. The identification and functional annotation of RNA structures conserved in vertebrates. Genome Res 27: 1371-1383. doi:10.1101/gr.208652.116

Smith MA, Gesell T, Stadler PF, Mattick JS. 2013. Widespread purifying selection on RNA structure in mammals. Nucleic Acids Res 41: 8220-8236. doi:10.1093/nar/gkt596

Trapnell C, Pachter L, Salzberg SL. 2009. TopHat: discovering splice junctions with RNA-seq. Bioinformatics 25: 1105-1111. doi:10 .1093/bioinformatics/btp120 
Trapnell C, Williams BA, Pertea G, Mortazavi A, Kwan G, Van Baren MJ, Salzberg SL, Wold BJ, Pachter L. 2010. Transcript assembly and quantification by RNA-seq reveals unannotated transcripts and isoform switching during cell differentiation. Nat Biotechnol 28: 511-515. doi:10.1038/nbt.1621

Ulitsky I. 2016. Evolution to the rescue: using comparative genomics to understand long non-coding RNAs. Nat Rev Genets 17: 601614. doi:10.1038/nrg.2016.85

Wang HV, Chekanova JA. 2017. Long noncoding RNAs in plants. Adv Exp Med Biol 1008: 133-154. doi:10.1007/978-981-105203-3_5

Wang Y, Fan X, Lin F, He G, Terzaghi W, Zhu D, Deng XW. 2014. Arabidopsis noncoding RNA mediates control of photomorphogenesis by red light. Proc Natl Acad Sci 111: 10359-10364. doi:10.1073/pnas.1409457111

Wang H, Niu OW, Wu HW, Liu J, Ye J, Yu N, Chua NH. 2015. Analysis of non-coding transcriptome in rice and maize uncovers roles of conserved IncRNAs associated with agriculture traits. Plant J 84: 404-416. doi:10.1111/tpj.13018

Wang D, Qu Z, Yang L, Zhang Q, Liu ZH, Do T, Adelson DL, Wang ZY, Searle I, Zhu JK. 2017. Transposable elements (TEs) contribute to stress-related long intergenic noncoding RNAs in plants. Plant $J$ 90: 133-146. doi:10.1111/tpj.13481

Washietl S, Hofacker IL, Stadler PF. 2005. Fast and reliable prediction of noncoding RNAs. Proc Natl Acad Sci 102: 2454-2459. doi:10 .1073/pnas.0409169102
Waterston RH, Lindblad-Toh K, Birney E, Rogers J, Abril JF, Agarwal P, Agarwala R, Ainscough R, Alexandersson M, An P, et al. 2002. Initial sequencing and comparative analysis of the mouse genome. Nature 420: 520-562. doi:10.1038/nature01262

Wheeler TJ, Eddy SR. 2013. nhmmer: DNA homology search with profile HMMs. Bioinformatics 29: 2487-2489. doi:10.1093/bioinfor matics/btt403

Yamada M. 2017. Functions of long intergenic non-coding (Linc) RNAs in plants. J Plant Res 130: 67-73. doi:10.1007/s10265016-0894-0

Yeo G, Burge CB. 2004. Maximum entropy modeling of short sequence motifs with applications to RNA splicing signals. J Comput Biol 11: 377-394. doi:10.1089/1066527041410418

Yu J, Tehrim S, Zhang F, Tong C, Huang J, Cheng X, Dong C, Zhou Y, Qin R, Hua W, et al. 2014. Genome-wide comparative analysis of NBS-encoding genes between Brassica species and Arabidopsis thaliana. BMC Genomics 15: 3. doi:10.1186/1471-2164-15-3

Yuan C, Meng X, Li X, Illing N, Ingle RA, Wang J, Chen M. 2017. PceRBase: a database of plant competing endogenous RNA. Nucleic Acids Res 45: D1009-D1014. doi:10.1093/nar/ gkw916

Zhang J, Wei L, Jiang J, Mason AS, Li H, Cui C, Chai L, Zheng B, Zhu Y, Xia Q, et al. 2018. Genome-wide identification, putative functionality and interactions between IncRNAs and miRNAs in Brassica species. Sci Rep 8: 4960. doi:10.1038/s41598-01823334-1 

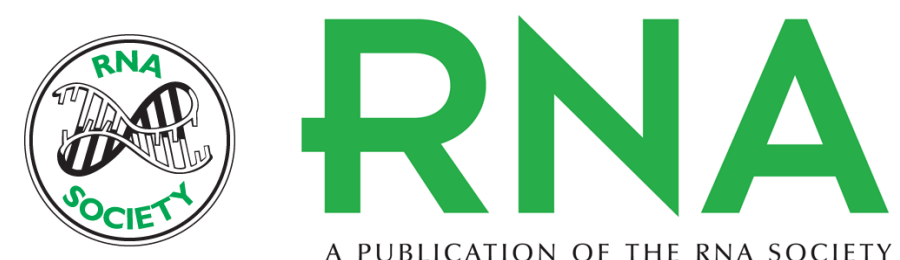

A PUBLICATION OF THE RNA SOCIETY

\section{Splicing conservation signals in plant long noncoding RNAs}

Jose Antonio Corona-Gomez, Irving Jair Garcia-Lopez, Peter F. Stadler, et al.

RNA 2020 26: 784-793 originally published online April 2, 2020

Access the most recent version at doi:10.1261/rna.074393.119

Supplemental Material

References

Creative Commons License

Email Alerting Service
http://rnajournal.cshlp.org/content/suppl/2020/04/02/rna.074393.119.DC1

This article cites 63 articles, 15 of which can be accessed free at: http://rnajournal.cshlp.org/content/26/7/784.full.html\#ref-list-1

This article is distributed exclusively by the RNA Society for the first 12 months after the full-issue publication date (see http://rnajournal.cshlp.org/site/misc/terms.xhtml). After 12 months, it is available under a Creative Commons License (Attribution-NonCommercial 4.0 International), as described at http://creativecommons.org/licenses/by-nc/4.0/.

Receive free email alerts when new articles cite this article - sign up in the box at the top right corner of the article or click here.

\section{|||||||| Providing Precise Solutions for} your research.

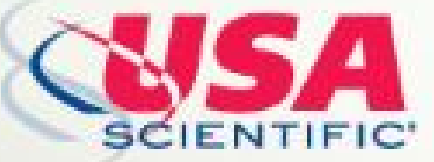

To subscribe to $R N A$ go to:

http://rnajournal.cshlp.org/subscriptions

(C) 2020 Corona-Gomez et al.; Published by Cold Spring Harbor Laboratory Press for the RNA Society 